\title{
Sufficient Conditions for Meromorphically $p$-Valent Starlikeness and Close-to-Convexity
}

\author{
B. A. Frasin, ${ }^{1}$ Tariq Al-Hawary, ${ }^{2}$ and M. Darus ${ }^{2}$ \\ ${ }^{1}$ Department of Mathematics, Faculty of Science, Al al-Bayt University, P.O. Box 130095, Mafraq, Jordan \\ ${ }^{2}$ School of Mathematical Sciences, Faculty of Science and Technology, Universiti Kebangsaan Malaysia, \\ 43600 Bangi, Selangor, Malaysia
}

Correspondence should be addressed to M. Darus; maslina@ukm.my

Received 14 October 2012; Accepted 6 December 2012

Academic Editor: JinLin Liu

Copyright (C) 2013 B. A. Frasin et al. This is an open access article distributed under the Creative Commons Attribution License, which permits unrestricted use, distribution, and reproduction in any medium, provided the original work is properly cited.

Making use of the linear operator $I_{\lambda}^{m}$ defined by (Frasin 2012), we introduce the class $\mathbb{M}_{p, j}^{m}(\lambda, \mu, \alpha)$ of meromorphically $p$-valent functions in the punctured unit disk $\mathscr{U}^{*}$. Furthermore, we obtain some sufficient conditions for starlikeness and close-to-convexity for functions belonging to this class. Several corollaries and consequences of the main results are also considered.

\section{Introduction and Definitions}

Let $\Sigma_{p, j}$ denote the class of functions of the form:

$$
f(z)=\frac{1}{z^{p}}+\sum_{n=j}^{\infty} a_{n+p-1} z^{n+p-1}, \quad(p, j \in \mathbb{N}:=\{1,2,3, \ldots\}),
$$

which are $p$-valent in the punctured unit disk $\mathscr{U}^{*}=\mathscr{U} \backslash\{0\}=$ $\{z: z \in \mathbb{C} ;|z|<1\}$. A function $f(z)$ in $\Sigma_{p, j}$ is said to be meromorphically $p$-valent starlike of order $\alpha$ if and only if

$$
\operatorname{Re}\left\{-\frac{z f^{\prime}(z)}{f(z)}\right\}>\alpha, \quad\left(z \in \mathcal{U}^{*}\right),
$$

for some $\alpha(0 \leq \alpha<p)$. We denote by $\Sigma_{p, j}^{*}(\alpha)$ the class of all meromorphically $p$-valent starlike of order $\delta$. Further, a function $f(z)$ in $\Sigma_{p, j}$ is said to be meromorphically $p$-valent convex of order $\alpha$ if and only if

$$
\operatorname{Re}\left\{-1-\frac{z f^{\prime \prime}(z)}{f^{\prime}(z)}\right\}>\alpha, \quad\left(z \in \mathscr{U}^{*}\right),
$$

for some $\alpha(0 \leq \alpha<p)$. We denote by $\Sigma_{p, j}^{k}(\alpha)$ the class of all meromorphically $p$-valent convex of order $\delta$. A function $f(z)$ belonging to $\Sigma_{p, j}$ is said to be meromorphically $p$-valent close-to-convex of order $\alpha$ if it satisfies

$$
\operatorname{Re}\left(-\frac{f^{\prime}(z)}{z^{-p-1}}\right)>\alpha, \quad\left(z \in \mathscr{U}^{*}\right),
$$

for some $\alpha(0 \leq \alpha<p)$. We denote by $\Sigma_{p, j}^{c}(\alpha)$ the subclass of $\Sigma_{p, j}$ consisting of functions which are meromorphically $p$ valent close-to-convex of order $\alpha$ in $\mathcal{U}^{*}$.

Many interesting families of analytic and multivalent functions were considered by earlier authors in Geometric Functions Theory (cf. e.g., [1-4]). Some subclasses of $\Sigma_{p, j}=$ $\Sigma$ when $p=j=1$ were considered by (e.g.) Miller [5], Pommerenke [6], Clunie [7], Owa et al. [8], and Royster [9]. Furthermore, several subclasses of $\Sigma_{p, j}=\Sigma_{p}$ when $j=1$ were studied by (amongst others) Mogra et al. [10], Uralegaddi and Ganigi [11], Cho et al. [12], Aouf [13, 14], and Uralegaddi and Somanatha [15].

For a function $f$ in $\Sigma_{p, j}$, Frasin [16] introduced and studied the following differential operator:

$$
\begin{gathered}
I^{0} f(z)=f(z), \\
I_{\lambda}^{1} f(z)=(1-\lambda) f(z)+\lambda z f^{\prime}(z)+\frac{\lambda(p+1)}{z^{p}}, \quad \lambda \geq 0, \\
I_{\lambda}^{2} f(z)=(1-\lambda) I_{\lambda}^{1} f(z)+\lambda z\left(I_{\lambda}^{1} f(z)\right)^{\prime}+\frac{\lambda(p+1)}{z^{p}},
\end{gathered}
$$


and for $m=1,2,3, \ldots$

$$
\begin{aligned}
I_{\lambda}^{m} f(z) & =(1-\lambda) I_{\lambda}^{m-1} f(z)+\lambda z\left(I_{\lambda}^{m-1} f(z)\right)^{\prime}+\frac{\lambda(p+1)}{z^{p}} \\
& =\frac{1}{z^{p}}+\sum_{n=j}^{\infty}[1+\lambda(p+n-2)]^{m} a_{n+p-1} z^{n+p-1} .
\end{aligned}
$$

Note that for $\lambda=p=j=1$, we have the operator $I^{m} f(z)$ introduced and studied by Frasin and Darus [17].

It easily verified from (6) that

$$
\begin{aligned}
& \lambda z\left(I_{\lambda}^{m} f(z)\right)^{\prime}=I_{\lambda}^{m+1} f(z)-(1-\lambda) I_{\lambda}^{m} f(z)-\frac{\lambda(p+1)}{z^{p}} \\
& \lambda z\left(I_{\lambda}^{m} f(z)\right)^{\prime \prime}=\left(I_{\lambda}^{m+1} f(z)\right)^{\prime}-\left(I_{\lambda}^{m} f(z)\right)^{\prime}+\frac{\lambda p(p+1)}{z^{p+1}} .
\end{aligned}
$$

Making use of the above operator $I_{\lambda}^{m}$, we now introduce a new class of meromorphically and $p$-valent functions defined as follows.

Definition 1. A function $f(z) \in \Sigma_{p, j}$ is said to be a member of the class $\mathbb{M}_{p, j}^{m}(\lambda, \mu, \alpha)$ if and only if

$$
\left|\frac{z^{p+1}\left(I_{\lambda}^{m} f(z)\right)^{\prime}}{\left(z^{p} I_{\lambda}^{m} f(z)\right)^{\mu-1}}+p\right|<p-\alpha,
$$

for some $\alpha(0 \leq \alpha<p) ; \mu \geq 0 ; \lambda \geq 0, p \in \mathbb{N}, m \in \mathbb{N}_{0}=$ $\mathbb{N} \cup\{0\}$ and for all $z \in \mathcal{U}^{*}$.

Note that condition (8) implies that

$$
\operatorname{Re}\left(-\frac{z^{p+1}\left(I_{\lambda}^{m} f(z)\right)^{\prime}}{\left(z^{p} I_{\lambda}^{m} f(z)\right)^{\mu-1}}\right)>\alpha
$$

Clearly, we have $\mathbb{M}_{p, j}^{0}(1,2, \alpha)=\Sigma_{p, j}^{*}(\alpha)$ and $\mathbb{M}_{p, j}^{0}(1$, $1, \alpha)=\Sigma_{p, j}^{c}(\alpha)$.

In this paper, we obtain some sufficient conditions for functions belonging to the class $\mathbb{M}_{p, j}^{m}(\lambda, \mu, \alpha)$. Several corollaries and consequences of the main results are also considered.

In order to derive our main results, we have to recall the following lemmas.

Lemma 2 (see [18]). Let $w(z)$ be analytic in $\mathcal{U}$ and such that $w(0)=0$. Then if $|w(z)|$ attains its maximum value on circle $|z|=r<1$ at a point $z_{o} \in \mathcal{U}$, we have

$$
z_{o} w^{\prime}\left(z_{o}\right)=k w\left(z_{o}\right)
$$

where $k \geq 1$ is a real number.

Lemma 3 (see [19]). Let $\Omega$ be a set in the complexplane $\mathbb{C}$ and suppose that $\Phi(z)$ is a mapping from $\mathbb{C}^{2} \times \mathcal{U}$ to $\mathbb{C}$ which satisfies $\Phi(i x, y ; z) \notin \Omega$ for $z \in \mathcal{U}$, and for all real $x, y$ such that $y \leq-n\left(1+x_{2}^{2}\right) / 2$. If the function $q(z)=1+q_{n} z^{n}+q_{n+1} z^{n+1}+\cdots$ is analytic in $\mathcal{U}$ such that $\Phi(q(z), z q(z) ; z) \in \Omega$ for all $z \in \mathcal{U}$, then $\operatorname{Re}\{q(z)\}>0$.

Lemma 4 (see [20]). Let $q(z)$ be analytic in $\mathcal{U}$ with $q(0)=1$. If there exists a point $z_{0} \in \mathcal{U}$ such that

$$
\begin{aligned}
& \operatorname{Re}\{q(z)\}>0, \quad\left(|z|<\left|z_{0}\right|\right), \\
& \operatorname{Re}\left\{q\left(z_{0}\right)\right\}=0, \quad q(z) \neq 0,
\end{aligned}
$$

then

$$
q\left(z_{0}\right)=i a, \quad \frac{z q^{\prime}\left(z_{0}\right)}{q\left(z_{0}\right)}=i \frac{k}{2}\left(a+\frac{1}{a}\right)
$$

where $a \in \mathbb{R} \backslash\{0\}$ and $k \geq 1$.

\section{Sufficient Conditions for Meromorphically $p$-Valent Starlikeness and Close-to-Convexity}

Making use of Lemma 2, we first prove

Theorem 5. If $f(z) \in \Sigma_{p, j}$ satisfies

$$
\begin{aligned}
\mid p & +1+\frac{1}{\lambda}\left[\frac{\left(I_{\lambda}^{m+1} f(z)\right)^{\prime}}{\left(I_{\lambda}^{m} f(z)\right)^{\prime}}-1+\frac{\lambda p(p+1)}{z^{p+1}\left(I_{\lambda}^{m} f(z)\right)^{\prime}}\right] \\
& -(\mu-1)\left[p+\frac{1}{\lambda}\left(\frac{I_{\lambda}^{m+1} f(z)}{I_{\lambda}^{m} f(z)}-(1-\lambda)-\frac{\lambda(p+1)}{z^{p} I_{\lambda}^{m} f(z)}\right)\right] \\
& -\gamma\left(\frac{z^{p+1}\left(I_{\lambda}^{m} f(z)\right)^{\prime}}{\left(z^{p} I_{\lambda}^{m} f(z)\right)^{\mu-1}}+p\right) \mid \\
& <\frac{(p-\alpha)(\gamma(2 p-\alpha)+1)}{2 p-\alpha}, \quad(z \in \mathcal{U}),
\end{aligned}
$$

for some $\alpha(0 \leq \alpha<p) ; \mu, \gamma \geq 0 ; \lambda>0, p \in \mathbb{N}$, and $m \in \mathbb{N}_{0}$, then $f(z) \in \mathbb{M}_{p, j}^{m}(\lambda, \mu, \alpha)$.

Proof. Define the function $w(z)$ by

$$
\frac{z^{p+1}\left(I_{\lambda}^{m} f(z)\right)^{\prime}}{\left(z^{p} I_{\lambda}^{m} f(z)\right)^{\mu-1}}=-p+(\alpha-p) w(z) .
$$


Then $w(z)$ is analytic in $\mathscr{U}$ and $w(0)=0$. It follows from (14) and the identities (7) that

$$
\begin{aligned}
p+1 & +\frac{1}{\lambda}\left[\frac{\left(I_{\lambda}^{m+1} f(z)\right)^{\prime}}{\left(I_{\lambda}^{m} f(z)\right)^{\prime}}-1+\frac{\lambda p(p+1)}{z^{p+1}\left(I_{\lambda}^{m} f(z)\right)^{\prime}}\right] \\
& -(\mu-1)\left[p+\frac{1}{\lambda}\left(\frac{I_{\lambda}^{m+1} f(z)}{I_{\lambda}^{m} f(z)}-(1-\lambda)-\frac{\lambda(p+1)}{z^{p} I_{\lambda}^{m} f(z)}\right)\right] \\
& -\gamma\left(\frac{z^{p+1}\left(I_{\lambda}^{m} f(z)\right)^{\prime}}{\left(z^{p} I_{\lambda}^{m} f(z)\right)^{\mu-1}}+p\right) \\
= & \gamma(p-\alpha) w(z)+\frac{(p-\alpha) z w^{\prime}(z)}{p+(p-\alpha) w(z)}
\end{aligned}
$$

Suppose that there exists $z_{0} \in \mathcal{U}$ such that

$$
\max _{|z|<z_{0}}|w(z)|=\left|w\left(z_{0}\right)\right|=1,
$$

then from Lemma 2, we have (10). Therefore, letting $z_{o} w^{\prime}\left(z_{o}\right)$ $=k e^{i \theta}(0 \leq \theta<2 \pi)$, with $k \geq 1$, we obtain that

$$
\begin{aligned}
\mid p & +1+\frac{1}{\lambda}\left[\frac{\left(I_{\lambda}^{m+1} f\left(z_{0}\right)\right)^{\prime}}{\left(I_{\lambda}^{m} f\left(z_{0}\right)\right)^{\prime}}-1+\frac{\lambda p(p+1)}{z_{0}^{p+1}\left(I_{\lambda}^{m} f\left(z_{0}\right)\right)^{\prime}}\right] \\
& -(\mu-1)\left[p+\frac{1}{\lambda}\left(\frac{I_{\lambda}^{m+1} f\left(z_{0}\right)}{I_{\lambda}^{m} f\left(z_{0}\right)}-(1-\lambda)-\frac{\lambda(p+1)}{z_{0}^{p} I_{\lambda}^{m} f\left(z_{0}\right)}\right)\right] \\
& -\gamma\left(\frac{z_{0}^{p+1}\left(I_{\lambda}^{m} f\left(z_{0}\right)\right)^{\prime}}{\left(z_{0}^{p} I_{\lambda}^{m} f\left(z_{0}\right)\right)^{\mu-1}}+p\right) \mid \\
& =\left|\gamma(p-\alpha) w\left(z_{0}\right)+\frac{(p-\alpha) z w^{\prime}\left(z_{0}\right)}{p+(p-\alpha) w\left(z_{0}\right)}\right| \\
& \geq \operatorname{Re}\left\{\gamma(p-\alpha)+\frac{(p-\alpha) k}{p+(p-\alpha) w\left(z_{0}\right)}\right\} \\
> & \gamma(p-\alpha)+\frac{p-\alpha}{2 p-\alpha}=\frac{(p-\alpha)(\gamma(2 p-\alpha)+1)}{2 p-\alpha},
\end{aligned}
$$

which contradicts our assumption (13). Therefore we have $|w(z)|<1$ in $\mathcal{U}$. Finally, we have

$$
\left|\frac{z^{p+1}\left(I_{\lambda}^{m} f(z)\right)^{\prime}}{\left(z^{p} I_{\lambda}^{m} f(z)\right)^{\mu-1}}+p\right|=(p-\alpha)|w(z)|<p-\alpha, \quad(z \in \mathcal{U}),
$$

that is, $f(z) \in \mathbb{M}_{p, j}^{m}(\lambda, \mu, \alpha)$. This completes the proof of the theorem.

Next we prove the following.
Theorem 6. If $f(z) \in \Sigma_{p, j}$ satisfies

$$
\begin{aligned}
& \operatorname{Re}\left\{\left(\frac{z^{p+1}\left(I_{\lambda}^{m} f(z)\right)^{\prime}}{\left(z^{p} I_{\lambda}^{m} f(z)\right)^{\mu-1}}\right)^{2}-\frac{z^{p+1}\left(I_{\lambda}^{m} f(z)\right)^{\prime}}{\left(z^{p} I_{\lambda}^{m} f(z)\right)^{\mu-1}}\right. \\
& \quad \times\left(1+\frac{1}{\lambda}\left[\frac{\left(I_{\lambda}^{m+1} f(z)\right)^{\prime}}{\left(I_{\lambda}^{m} f(z)\right)^{\prime}}-1+\frac{\lambda p(p+1)}{z^{p+1}\left(I_{\lambda}^{m} f(z)\right)^{\prime}}\right]\right. \\
& \left.\left.+\frac{1-\mu}{\lambda}\left[\frac{I_{\lambda}^{m+1} f(z)}{I_{\lambda}^{m} f(z)}-\frac{\lambda(p+1)}{z^{p} I_{\lambda}^{m} f(z)}\right]\right)\right\} \\
& >\delta\left(\delta+\frac{(1-\mu)(1-\lambda)}{\lambda}+\frac{n}{2}\right)+p\left(\delta(\mu-2)-\frac{n}{2}\right),
\end{aligned}
$$

for some $\delta(0 \leq \delta<p) ; \mu \geq 0 ; \lambda>0, p \in \mathbb{N}$ and $m \in$ $\mathbb{N}_{0}$, then $f(z) \in \mathbb{M}_{p, j}^{m}(\lambda, \mu, \delta)$.

Proof. Define the function $q(z)$ by

$$
\frac{z^{p+1}\left(I_{\lambda}^{m} f(z)\right)^{\prime}}{\left(z^{p} I_{\lambda}^{m} f(z)\right)^{\mu-1}}=-\delta+(\delta-p) q(z)
$$

Then, we see that $q(z)=1+q_{n} z^{n}+q_{n+1} z^{n+1}+\cdots$ is analytic in $\mathcal{U}$. Differentiating both sides of (20) with respect $z$ logarithmically, we get

$$
\begin{gathered}
1+\frac{z\left(I_{\lambda}^{m} f(z)\right)^{\prime \prime}}{\left(I_{\lambda}^{m} f(z)\right)^{\prime}}+(1-\mu) \frac{z\left(I_{\lambda}^{m} f(z)\right)^{\prime}}{I_{\lambda}^{m} f(z)} \\
=\frac{(p-\delta) z q^{\prime}(z)}{\delta+(p-\delta) q(z)}+p(\mu-2) .
\end{gathered}
$$

Using the identities (7) in (21), we find that

$$
\begin{aligned}
1+\frac{1}{\lambda}\left[\frac{\left(I_{\lambda}^{m+1} f(z)\right)^{\prime}}{\left(I_{\lambda}^{m} f(z)\right)^{\prime}}-1+\frac{\lambda p(p+1)}{z^{p+1}\left(I_{\lambda}^{m} f(z)\right)^{\prime}}\right] \\
+\frac{1-\mu}{\lambda}\left[\frac{I_{\lambda}^{m+1} f(z)}{I_{\lambda}^{m} f(z)}-\frac{\lambda(p+1)}{z^{p} I_{\lambda}^{m} f(z)}\right] \\
=\frac{(p-\delta) z q^{\prime}(z)}{\delta+(p-\delta) q(z)}+p(\mu-2)+\frac{(1-\mu)(1-\lambda)}{\lambda} .
\end{aligned}
$$


From (20) and (22), we immediately get

$$
\begin{aligned}
& \left(\frac{z^{p+1}\left(I_{\lambda}^{m} f(z)\right)^{\prime}}{\left(z^{p} I_{\lambda}^{m} f(z)\right)^{\mu-1}}\right)^{2}-\frac{z^{p+1}\left(I_{\lambda}^{m} f(z)\right)^{\prime}}{\left(z^{p} I_{\lambda}^{m} f(z)\right)^{\mu-1}} \\
& \quad \times\left(1+\frac{1}{\lambda}\left[\frac{\left(I_{\lambda}^{m+1} f(z)\right)^{\prime}}{\left(I_{\lambda}^{m} f(z)\right)^{\prime}}-1+\frac{\lambda p(p+1)}{z^{p+1}\left(I_{\lambda}^{m} f(z)\right)^{\prime}}\right]\right. \\
& =(p-\delta) z q^{\prime}(z)+(p-\delta)^{2} q^{2}(z)+(p-\delta) q(z) \\
& \quad+\left(p(\mu-2)+\frac{(1-\mu)(1-\lambda)}{\lambda}+2 \delta\right) \\
& +\left(p(\mu-2)+\frac{(1-\mu)(1-\lambda)}{\lambda}\right) \delta+\delta_{\lambda}^{m+1} f(z) \\
& \left.\quad\left(p I_{\lambda}^{m} f(z)\right]\right) \\
& = \\
& \quad \Phi\left(q(z), z q^{\prime}(z) ; z\right),
\end{aligned}
$$

where

$$
\begin{aligned}
\Phi(r, s ; t)= & (p-\delta) s+(p-\delta)^{2} r^{2} \\
& +(p-\delta) r\left(p(\mu-2)+\frac{(1-\mu)(1-\lambda)}{\lambda}+2 \delta\right) \\
& +\left(p(\mu-2)+\frac{(1-\mu)(1-\lambda)}{\lambda}\right) \delta+\delta^{2} .
\end{aligned}
$$

For all real $x, y$ satisfying $y \leq-n\left(1+x_{2}^{2}\right) / 2$, we have

$$
\begin{aligned}
\operatorname{Re} \Phi(i x, y ; z)= & (p-\delta) y-(p-\delta)^{2} x^{2} \\
& +\left(p(\mu-2)+\frac{(1-\mu)(1-\lambda)}{\lambda}\right) \delta+\delta^{2} \\
\leq & -\frac{n}{2}(p-\delta)-(p-\delta)\left[\frac{n}{2}+p-\delta\right] x^{2} \\
& +\left(p(\mu-2)+\frac{(1-\mu)(1-\lambda)}{\lambda}\right) \delta+\delta^{2} \\
\leq & \left(p(\mu-2)+\frac{(1-\mu)(1-\lambda)}{\lambda}\right) \delta+\delta^{2}-\frac{n}{2}(p-\delta) \\
= & \delta\left(\delta+\frac{(1-\mu)(1-\lambda)}{\lambda}+\frac{n}{2}\right)+p\left(\delta(\mu-2)-\frac{n}{2}\right) .
\end{aligned}
$$

Let $\Omega=\{w: \operatorname{Re} w>\delta(\delta+(1-\mu)(1-\lambda) / \lambda+n / 2)+p(\delta(\mu-$ $2)-n / 2)\}$. Then $\Phi\left(q(z), z q^{\prime}(z) ; z\right) \in \Omega$, and $\Phi(i x, y ; z) \notin \Omega$, for all real $x$ and $y \leq-n\left(1+x_{2}^{2}\right) / 2, z \in \mathcal{U}$. By using Lemma 3 , we have $\operatorname{Re} q(z)>0$, that is, $f(z) \in \mathbb{M}_{p, j}^{m}(\lambda, \mu, \delta)$.
Finally, we prove the next theorem.

Theorem 7. If $f(z) \in \Sigma_{p, j}$ satisfies

$$
\begin{aligned}
\operatorname{Re}\{ & (\mu-1)\left(\frac{I_{\lambda}^{m+1} f(z)}{I_{\lambda}^{m} f(z)}-\frac{\lambda(p+1)}{z^{p} I_{\lambda}^{m} f(z)}\right) \\
& \left.-\left(\frac{\left(I_{\lambda}^{m+1} f(z)\right)^{\prime}}{\left(I_{\lambda}^{m} f(z)\right)^{\prime}}-1+\frac{\lambda p(p+1)}{z^{p+1}\left(I_{\lambda}^{m} f(z)\right)^{\prime}}\right)\right\} \\
< & \lambda-p \lambda(\mu-2)-(1-\mu)(1-\lambda)+\frac{\lambda(p-\delta)}{2 \delta}
\end{aligned}
$$

for some $\delta(p / 2 \leq \delta<p) ; \mu \geq 0 ; \lambda>0, p \in \mathbb{N}$, and $m \in \mathbb{N}_{0}$, then $f(z) \in \mathbb{M}_{p, j}^{m}(\lambda, \mu, \delta)$.

Proof. Define the function $q(z)$ by

$$
-\frac{z^{p+1}\left(I_{\lambda}^{m} f(z)\right)^{\prime}}{\left(z^{p} I_{\lambda}^{m} f(z)\right)^{\mu-1}}=\delta+(p-\delta) q(z) .
$$

Then, we see that $q(z)$ is analytic in $\mathscr{U}$ with $q(0)=1$. From (22) it follows that and

$$
\begin{aligned}
(\mu-1) & \left(\frac{I_{\lambda}^{m+1} f(z)}{I_{\lambda}^{m} f(z)}-\frac{\lambda(p+1)}{z^{p} I_{\lambda}^{m} f(z)}\right) \\
& -\left(\frac{\left(I_{\lambda}^{m+1} f(z)\right)^{\prime}}{\left(I_{\lambda}^{m} f(z)\right)^{\prime}}-1+\frac{\lambda p(p+1)}{z^{p+1}\left(I_{\lambda}^{m} f(z)\right)^{\prime}}\right) \\
= & \lambda-p \lambda(\mu-2)-(1-\mu)(1-\lambda)-\frac{\lambda(p-\delta) z q^{\prime}(z)}{\delta+(p-\delta) q(z)}
\end{aligned}
$$

If there exists a point $z_{0} \in \mathcal{U}$ such that

$$
\begin{aligned}
& \operatorname{Re}\{q(z)\}>0, \quad\left(|z|<\left|z_{0}\right|\right), \\
& \operatorname{Re}\left\{q\left(z_{0}\right)\right\}=0, \quad q(z) \neq 0 .
\end{aligned}
$$

Then applying Lemma 4, we have

$$
q\left(z_{0}\right)=i a, \quad \frac{z q^{\prime}\left(z_{0}\right)}{q\left(z_{0}\right)}=i \frac{k}{2}\left(a+\frac{1}{a}\right)
$$


where $a \in \mathbb{R} \backslash\{0\}$ and $k \geq 1$. Thus, from (28) and (30) we get

$$
\begin{aligned}
(\mu-1) & \left(\frac{I_{\lambda}^{m+1} f\left(z_{0}\right)}{I_{\lambda}^{m} f\left(z_{0}\right)}-\frac{\lambda(p+1)}{z_{0}^{p} I_{\lambda}^{m} f\left(z_{0}\right)}\right) \\
& -\left(\frac{\left(I_{\lambda}^{m+1} f\left(z_{0}\right)\right)^{\prime}}{\left(I_{\lambda}^{m} f\left(z_{0}\right)\right)^{\prime}}-1+\frac{\lambda p(p+1)}{z_{0}^{p+1}\left(I_{\lambda}^{m} f\left(z_{0}\right)\right)^{\prime}}\right) \\
= & \lambda-p \lambda(\mu-2)-(1-\mu)(1-\lambda)-\frac{\lambda(p-\delta) z_{0} q^{\prime}\left(z_{0}\right)}{\delta+(p-\delta) q\left(z_{0}\right)} \\
= & \lambda-p \lambda(\mu-2)-(1-\mu)(1-\lambda)+\frac{k \lambda(p-\delta)\left(1+a^{2}\right)}{2(\delta+i(p-\delta) a)} .
\end{aligned}
$$

Therefore, we have

$$
\begin{aligned}
\operatorname{Re}\left\{(\mu-1)\left(\frac{I_{\lambda}^{m+1} f\left(z_{0}\right)}{I_{\lambda}^{m} f\left(z_{0}\right)}-\frac{\lambda(p+1)}{z_{0}^{p} I_{\lambda}^{m} f\left(z_{0}\right)}\right)\right. \\
\left.\quad-\left(\frac{\left(I_{\lambda}^{m+1} f\left(z_{0}\right)\right)^{\prime}}{\left(I_{\lambda}^{m} f\left(z_{0}\right)\right)^{\prime}}-1+\frac{\lambda p(p+1)}{z_{0}^{p+1}\left(I_{\lambda}^{m} f\left(z_{0}\right)\right)^{\prime}}\right)\right\} \\
=\lambda-p \lambda(\mu-2)-(1-\mu)(1-\lambda)+\frac{k \lambda(p-\delta)\left(1+a^{2}\right) \delta}{2\left(\delta^{2}+(p-\delta)^{2} a^{2}\right)} \\
\geq \lambda-p \lambda(\mu-2)-(1-\mu)(1-\lambda)+\frac{k \lambda(p-\delta)}{2 \delta} \\
\geq \lambda-p \lambda(\mu-2)-(1-\mu)(1-\lambda)+\frac{\lambda(p-\delta)}{2 \delta} .
\end{aligned}
$$

This contradicts our assumption. Thus, we conclude that $\operatorname{Re} q(z)>0$ for all $z \in \mathscr{U}$, that is,

$$
\operatorname{Re}\left(-\frac{z^{p+1}\left(I_{\lambda}^{m} f(z)\right)^{\prime}}{\left(z^{p} I_{\lambda}^{m} f(z)\right)^{\mu-1}}\right)>\delta .
$$

\section{Special Cases and Consequences}

Among the various interesting and important consequences of Theorems 5-7, we mention now some of the corollaries relating to the classes $\Sigma_{p, j}^{*}(\alpha)$, and $\Sigma_{p, j}^{c}(\delta)$, which are deducible from the main results.

Firstly, if we let $m=0, \mu=2$, and $\lambda=1$ in Theorems 5-7, we get the following sufficient conditions for meromorphically $p$-valent starlike functions.

Corollary 8. If $f(z) \in \Sigma_{p, j}$ satisfies

$$
\begin{aligned}
\mid 1 & +\frac{z f^{\prime \prime}(z)}{f^{\prime}(z)}-(1+\gamma) \frac{z f^{\prime}(z)}{f(z)}-\gamma p \mid \\
& <\frac{(p-\alpha)(\gamma(2 p-\alpha)+1)}{2 p-\alpha},
\end{aligned}
$$

for some $\alpha(0 \leq \alpha<p) ; p \in \mathbb{N}$ and $\gamma \geq 0$, then $f(z) \in$ $\Sigma_{p, j}^{*}(\alpha)$.

Corollary 9. If $f(z) \in \Sigma_{p, j}$ satisfies

$$
\begin{aligned}
& \operatorname{Re}\left\{\left(\frac{z f^{\prime}(z)}{f(z)}\right)\left(\frac{2 z f^{\prime}(z)}{f(z)}-1-\frac{z f^{\prime \prime}(z)}{f^{\prime}(z)}\right)\right\} \\
& >\delta\left(\delta+\frac{n}{2}\right)-\frac{n p}{2},
\end{aligned}
$$

for some $\delta(0 \leq \delta<p) ; p \in \mathbb{N}$ and $\gamma \geq 0$, then $f(z) \in$ $\Sigma_{p, j}^{*}(\delta)$.

Corollary 10. If $f(z) \in \Sigma_{p, j}$ satisfies

$$
\operatorname{Re}\left\{\frac{z f^{\prime}(z)}{f(z)}-\frac{z f^{\prime \prime}(z)}{f^{\prime}(z)}\right\}<1+\frac{p-\delta}{2 \delta},
$$

for some $\delta(p / 2 \leq \delta<p) ; p \in \mathbb{N}$, then $f(z) \in \Sigma_{p, j}^{*}(\delta)$.

Setting $m=0$ and $\mu=\lambda=1$ in Theorems 5-7, we get the following sufficient conditions for meromorphically $p$-valent close-to-convex functions.

Corollary 11. If $f(z) \in \Sigma_{p, j}$ satisfies

$$
\begin{gathered}
\left|p+1+\frac{z f^{\prime \prime}(z)}{f^{\prime}(z)}-\gamma\left(z^{p+1} f^{\prime}(z)+p\right)\right| \\
<\frac{(p-\alpha)(\gamma(2 p-\alpha)+1)}{2 p-\alpha},
\end{gathered}
$$

for some $\alpha(0 \leq \alpha<p) ; p \in \mathbb{N}$ and $\gamma \geq 0$, then $f(z) \in$ $\Sigma_{p, j}^{c}(\alpha)$.

Corollary 12. If $f(z) \in \Sigma_{p, j}$ satisfies

$$
\begin{aligned}
& \operatorname{Re}\left\{\left(z^{p+1} f^{\prime}(z)\right)\left(z^{p+1} f^{\prime}(z)-1-\frac{z f^{\prime \prime}(z)}{f^{\prime}(z)}\right)\right\} \\
& >(\delta-p)\left(\delta+\frac{n}{2}\right),
\end{aligned}
$$

for some $\delta(0 \leq \delta<p) ; p \in \mathbb{N}$, then $f(z) \in \Sigma_{p, j}^{c}(\delta)$.

Corollary 13. If $f(z) \in \Sigma_{p, j}$ satisfies

$$
\operatorname{Re}\left\{-\frac{z f^{\prime \prime}(z)}{f^{\prime}(z)}\right\}<1+p+\frac{p-\delta}{2 \delta},
$$

for some $\delta(p / 2 \leq \delta<p) ; p \in \mathbb{N}$, then $f(z) \in \Sigma_{p, j}^{c}(\delta)$.

Setting $p=j=1$ in Corollary 10, we have

Corollary 14. If $f(z) \in \Sigma$ satisfies

$$
\operatorname{Re}\left\{\frac{z f^{\prime}(z)}{f(z)}-\frac{z f^{\prime \prime}(z)}{f^{\prime}(z)}\right\}<1+\frac{1-\delta}{2 \delta},
$$


for some $\delta(1 / 2 \leq \delta<1)$, then $f(z) \in \Sigma^{*}(\delta)$. In particular, if $f(z) \in \sum$ satisfies

$$
\operatorname{Re}\left\{\frac{z f^{\prime}(z)}{f(z)}-\frac{z f^{\prime \prime}(z)}{f^{\prime}(z)}\right\}<\frac{3}{2},
$$

then $f(z)$ is meromorphically starlike of order $1 / 2$.

Setting $p=j=1$ in Corollary 13, we have the following.

Corollary 15. If $f(z) \in \Sigma$ satisfies

$$
\operatorname{Re}\left\{-\frac{z f^{\prime \prime}(z)}{f^{\prime}(z)}\right\}<2+\frac{1-\delta}{2 \delta},
$$

for some $\delta(1 / 2 \leq \delta<1)$, then $f(z) \in \Sigma^{c}(\delta)$. In particular, if $f(z) \in \Sigma$ satisfies

$$
\operatorname{Re}\left\{-\frac{z f^{\prime \prime}(z)}{f^{\prime}(z)}\right\}<\frac{5}{2},
$$

then $f(z)$ is meromorphically close-to-convex of order $1 / 2$.

Remark 16. (i) If we put $\gamma=p=j=1$ in Corollaries 8 and 11, we get Corollaries 5 and 1, respectively, proved by Goyal and Prajapat [21].

(ii) If we put $p=j=n=1$ and $\delta=0$ in Corollaries 9 and 12 , we get Corollaries 8 and 4 , respectively, proved by Goyal and Prajapat [21].

\section{Acknowledgment}

The work here was fully supported by LRGS/TD/2011/UKM/ ICT/03/02.

\section{References}

[1] M. K. Aouf, "A generalization of meromorphic multivalent functions with positive coefficients," Mathematica Japonica, vol. 35, no. 4, pp. 609-614, 1990.

[2] S. R. Kulkarni, U. H. Naik, and H. M. Srivastava, "A certain class of meromorphically $p$-valent quasi-convex functions," Panamerican Mathematical Journal, vol. 8, no. 1, pp. 57-64, 1998.

[3] H. M. Srivastava and S. Owa, Current Topics in Analytic Function Theory, World Scientific Publishing Co, River Edge, NJ, USA, 1992.

[4] H. M. Srivastava, H. M. Hossen, and M. K. Aouf, "A unified presentation of some classes of meromorphically multivalent functions," Computers \& Mathematics with Applications, vol. 38, no. 11-12, pp. 63-70, 1999.

[5] J. Miller, "Convex meromorphic mappings and related functions," Proceedings of the American Mathematical Society, vol. 25, pp. 220-228, 1970.

[6] Ch. Pommerenke, "On meromorphic starlike functions," Pacific Journal of Mathematics, vol. 13, pp. 221-235, 1963.

[7] J. Clunie, "On meromorphic schlicht functions," Journal of the London Mathematical Society, vol. 34, pp. 215-216, 1959.
[8] S. Owa, H. E. Darwish, and M. K. Aouf, "Meromorphic multivalent functions with positive and fixed second coefficients," Mathematica Japonica, vol. 46, no. 2, pp. 231-236, 1997.

[9] W. C. Royster, "Meromorphic starlike multivalent functions," Transactions of the American Mathematical Society, vol. 107, pp. 300-308, 1963.

[10] M. L. Mogra, T. R. Reddy, and O. P. Juneja, "Meromorphic univalent functions with positive coefficients," Bulletin of the Australian Mathematical Society, vol. 32, no. 2, pp. 161-176, 1985.

[11] B. A. Uralegaddi and M. D. Ganigi, "A certain class of meromorphically starlike functions with positive coefficients," Pure and Applied Mathematika Sciences, vol. 26, no. 1-2, pp. 75-81, 1987.

[12] N. E. Cho, S. H. Lee, and S. Owa, "A class of meromorphic univalent functions with positive coefficients," Kobe Journal of Mathematics, vol. 4, no. 1, pp. 43-50, 1987.

[13] M. K. Aouf, "On a class of meromorphic multivalent functions with positive coefficients," Mathematica Japonica, vol. 35, no. 4, pp. 603-608, 1990.

[14] M. K. Aouf, "On a certain class of meromorphic univalent functions with positive coefficients," Rendiconti di Matematica e delle sue Applicazioni Serie VII, vol. 11, no. 2, pp. 209-219, 1991.

[15] B. A. Uralegaddi and C. Somanatha, "New criteria for meromorphic starlike univalent functions," Bulletin of the Australian Mathematical Society, vol. 43, no. 1, pp. 137-140, 1991.

[16] B. A. Frasin, "Differential operator of meromorphic p-valent functions," Tamkang Journal of Mathematics, vol. 43, pp. 417-421, 2012.

[17] B. A. Frasin and M. Darus, "On certain meromorphic functions with positive coefficients," Southeast Asian Bulletin of Mathematics, vol. 28, no. 4, pp. 615-623, 2004.

[18] I. S. Jack, "Functions starlike and convex of order $\alpha$," Journal of the London Mathematical Society, vol. 3, pp. 469-474, 1971.

[19] S. S. Miller and P. T. Mocanu, "Differential subordinations and inequalities in the complex plane," Journal of Differential Equations, vol. 67, no. 2, pp. 199-211, 1987.

[20] M. Nunokawa, "On properties of non-Carathéodory functions," Proceedings of the Japan Academy, Series A, vol. 68, no. 6, pp. 152-153, 1992.

[21] S. P. Goyal and J. K. Prajapat, "A new class of meromorphic multivalent functions involving certain linear operator," Tamsui Oxford Journal of Mathematical Sciences, vol. 25, no. 2, pp. 167-176, 2009. 


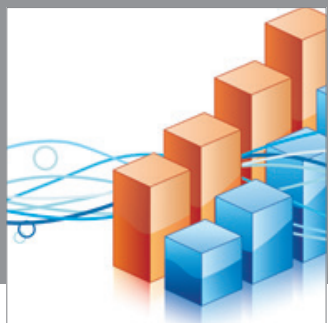

Advances in

Operations Research

mansans

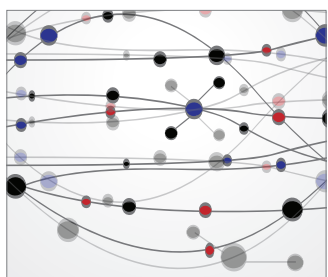

The Scientific World Journal
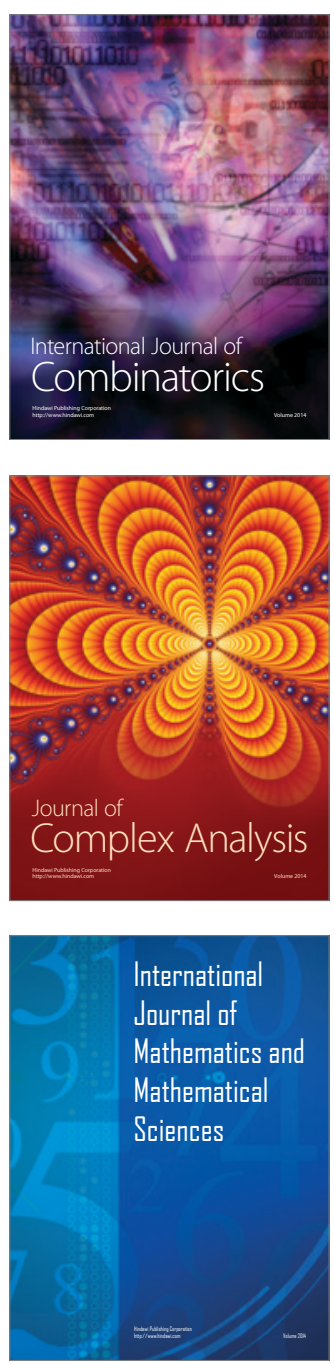
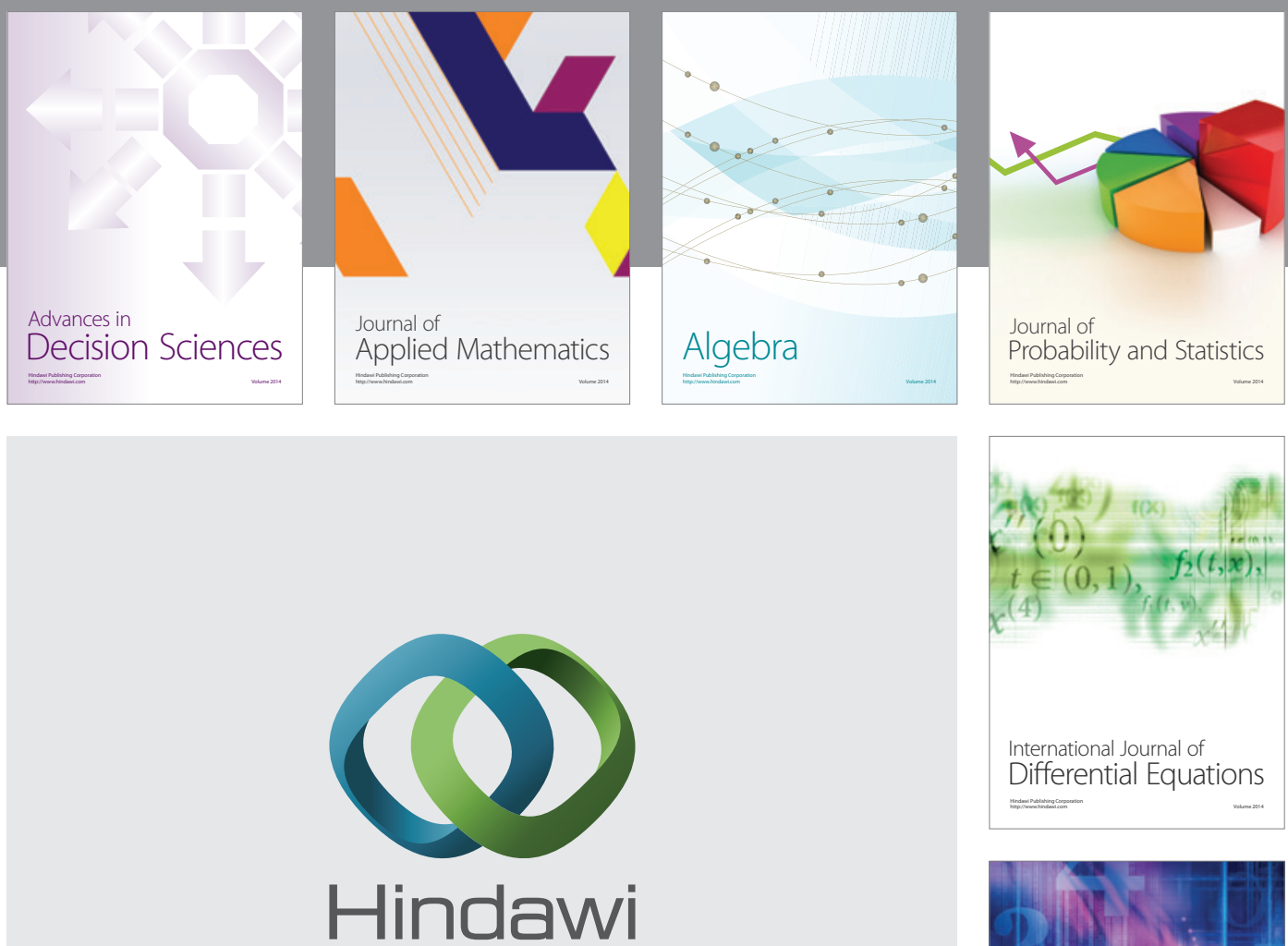

Submit your manuscripts at http://www.hindawi.com
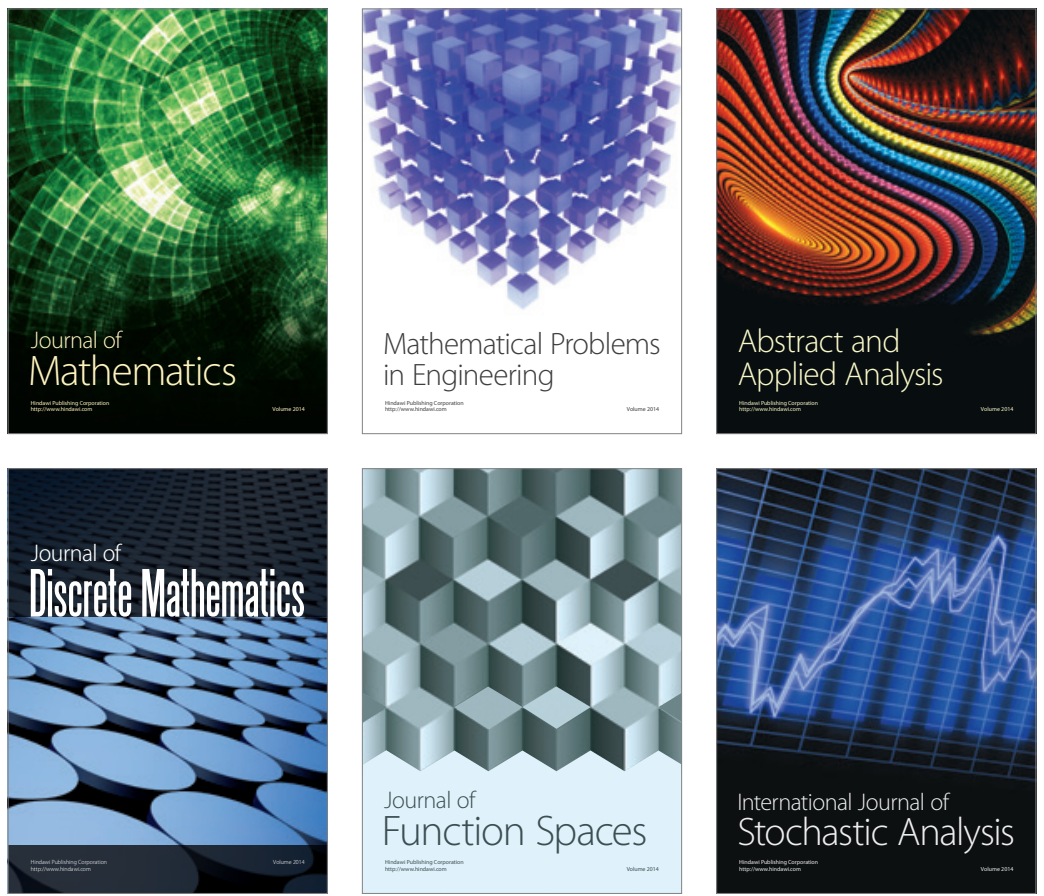

Journal of

Function Spaces

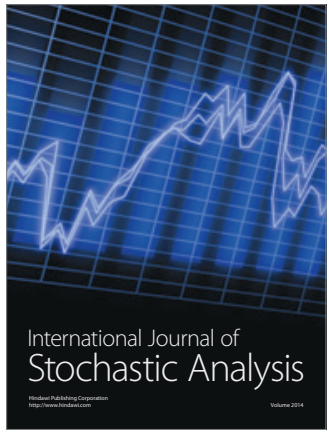

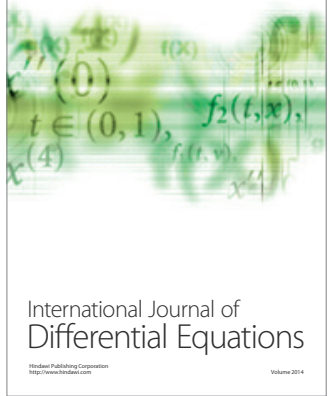
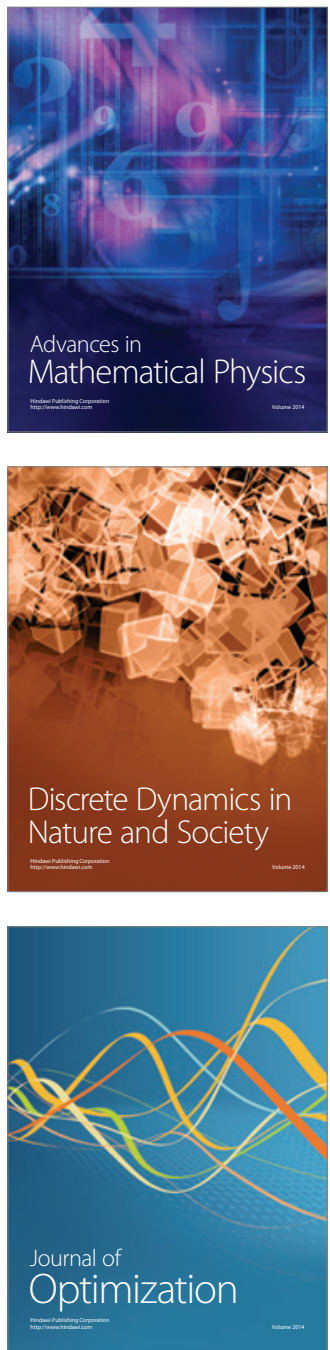